\title{
Energy Attentive and Pre-Fault Recognize Mechanism for Distributed Wireless Sensor Network Using Fuzzy Logic Approach
}

Roshani Talmale ( $\square$ roshanikambe2011@gmail.com )

Vignan's Foundation for Science Technology and Research Nirupama Bhat

Vignan's Foundation for Science Technology and Research

\section{Research Article}

Keywords: Fuzzy rule set, fault count, faulty node, energy efficiency, wireless sensor network.

Posted Date: June 4th, 2021

DOl: https://doi.org/10.21203/rs.3.rs-226503/v1

License: (c) (i) This work is licensed under a Creative Commons Attribution 4.0 International License. Read Full License

Version of Record: A version of this preprint was published at Wireless Personal Communications on December 1st, 2021. See the published version at https://doi.org/10.1007/s11277-021-09405-z. 


\title{
Energy attentive and Pre-Fault Recognize Mechanism for Distributed Wireless Sensor Network Using Fuzzy Logic approach
}

\author{
${ }^{1}$ Roshani Talmale , \\ VFSTR, Vignan's University, Vadalamudi, AP, India \\ roshanikambe2011@gmail.com \\ ${ }^{2}$ M.Nirupama Bhat, \\ Vignan's University, VFSTR, Deemed to be University, Vadalamudi, AP, India \\ mundukur@gmail.com
}

\begin{abstract}
Wireless Sensor Network (WSN) have transformed over recent years with incredible development in design of smart real time applications. In spite of countless potential, it poses numerous design issues like fault tolerant communication, low latency, scalability, transmission media and coverage problems. One of the foremost design issues of WSN is to detect the run time fault because it is unaware of internal operation of the sensor node. As a result, valuable sensed information is unable to reach its destination and performance start degrading throughout the routing period of the network. To address this issue, the proposed mechanism design a novel Pre-fault detection mechanism based on fuzzy rule based mechanism for multilevel transmission in distributed sensor networks. Many researchers have been focused on fault detection mechanisms without considering the dynamic behavior status of sensor node. In this proposed mechanism the Fuzzy decision rule set is identified to take routing decisions based on fuzzy fault count status. This mechanism helps to recognize the fault in advance and a routing decision has been taken in order to save energy as well as minimize delay. On the basis of node fault status data transmission rate is finalized in order to avoid more energy depletion. The experimented simulations results prove the accuracy of the proposed mechanism. The results demonstrate that the proposed mechanism performed well on judgment evaluation metrics such as energy dissipation ratio, packet drop rate and communication delay.
\end{abstract}

2015 Published by Elsevier Ltd.

\section{KEYWORDS:}

Fuzzy rule set, fault count, faulty node, energy efficiency, wireless sensor network.

\section{Introduction}

The Wireless Sensor Network is having a selfstructured setup and deploy in a hostile environment where human intervention is almost negligible. In order to get better results from the sensor network, the sensor node is required to work for a longer time and should maintain network topology. The lifespan of a sensor network is influenced by well-organized use of the accessible resources, particularly battery capacity of sensor node [1]. Minimizing the battery consumption is a major challenge of wireless sensor networks. Another significant challenge of WSNs is to design the fault tolerant routing technique. Sensor nodes are susceptible to failures because of several environmental threats. To enhance the performance of WSN it is necessary to discover the defective nodes and recover them immediately to maintain network connections. The performance measurable parameter of sensor network and Quality of Service (QoS) is depends on faulty node discovery and recovery mechanism within the stipulated time period. Quality of Services (QoS) of the network continuously degrades if sensor faults cannot be controlled [2].

Wireless Sensor Network (WSNs) has been proved to be a prominent technology for designing and deploying real time applications due to its vast prospective to monitor the real world. Sensor nodes are operational with a gathering of wireless radio transducer, supervisor unit and storage unit, which are deployed in distributed and dynamically [3]. Due to fast development in Micro-electronic devices it is credible to design smart sensors with better enhancement, accuracy and support extensive real time application like target following, natural disaster management, unsafe atmosphere and seismic recognizing, etc. [4].

Instant faults identification in the data routing process due to unstructured and unpredictable behaviour is very crucial task in WSNs. Furthermore, most of the existing fault detection methods in WSNs detect only permanent faults during the fault diagnosis periods. Sensor node forward the data to other sensor nodes those who are in transmission range, if acknowledge is not received then permanent fault is detected. But energy is lost to transfer the data to the failure node. We can restrict this using a pre fault detection method. Before transmitting the data, check the energy level of the next node, if it is below threshold value then stop the transmission because that node is not able to process the data [5]. This mechanism is used to save energy. The proposed study is based on a fuzzy fault 
classification and maintenance scheme for WSNs that can detect permanent fault in case of low energy, transit fault and stuck in fault based on sensing information in network and save the energy by performing operations according to their energy status. In order to improve the result of wireless sensor networks, fuzzy logic decision-based mechanisms are useful.

The use of fuzzy logic in WSNs is shown to be a promising technique since it permits integration of diverse parameters in an efficient manner and produces realistic figures [6][7]. Fuzzy logic approach is well-matched for sensor functionality. The potential of fuzzy logic goes beyond the expectation of modern systems and can be used in many research domains. In WSNs, fuzzy logic has been used to make decisions in routing data in case of fault. It helps to reduce power consumption, and rapidly enhances performance of the network [9].

The major contributions of the proposed mechanism can be listed as follows:

- Presenting a novel distributed fuzzy logic approach to detect the faulty node in the target monitoring area and categories them in three states.

- The proposed mechanism is distributed, it uses the node in data routing process as per their computing status capabilities.

- Enhances the detection accuracy of the faults in the data routing process.

- Comprehensive simulations are performed in various wireless scenarios.

\section{Related Work}

In this section, the existing research based on fault detection using fuzzy logic systems is discussed. Saeed Javanmard et al. [10] have proposed faulty node detection using fuzzy interpretation attributes. It predicts the ratio of faulty nodes using voting technique. The routing protocol suggested by the author is focused on detecting faults at intermediate nodes also. The parameters which are considered for lifetime expansion are records processing complexity, point to point delay and energy consumption in WSN.

Fault detection pattern for failure nodes in wireless sensor networks. The simulation model defines the performance of the distributed fault detection method for fault detection accuracy greatly. In this prototype, each sensor node periodically communicates with a neighboring node by sending a beacon message to know the status of the active node. Every sensor node retains the neighbor node information's current energy level, bandwidth, and link competence statistics between the nodes; based on these collected parameter values each node detects the faulty nodes within its transmission range by using fuzzy logic estimate approach.

Sasmita Acharyaet. al. [12] have proposed a deterministic cluster deployment model fuzzy optimization model. Faulty nodes are identified by a data aggregation process. Fuzzy estimator is used to discover intra-cluster and inter-cluster faults in WSNs for diverse fault categories. This algorithm calculates the Fault index for each sensor and forward the value to the respective $\mathrm{CH}$. Clusters head comparing their index value with the predefined threshold index value. If the CFI measures is less than the defined threshold value, then the cluster is identified as a standard cluster, otherwise it is considered a faulty node and circulates this information to the neighbor's node. Fault tolerance mechanism using fuzzy knowledge is discussed in (Sasmita et al., 2018). The proposed study is using a sleep state mechanism to conserve the energy and fuzzy rule to detect fault in network [13] .Prasenjit et.al. (2016) proposed a fuzzy rule-based fault detection mechanism for scalable network. Grouping and supervision of faulty nodes is none depending on their fault status. Due to uncertainties in the WSN environment, a fuzzy logic approach is utilized to get better results. It categorizes different nodes according to the set of functions and creates a non-fuzzy control to acquire proper sensed value from various types of nodes [14].

Raghunath ,Thirukumaran et al., (2019) have implemented instant synchronization by finding faulty nodes. This approach is also using a fuzzy based estimator to identify faults in the routing process. It avoids collision and reduced delay in communication. It implements a rapid installation system using fuzzy support approach [15]. FarnazPakdelet.al.(2016) proposed cluster head fault detection using fuzzy methods. In this approach cluster head fault is detected if no response is received in a particular time period. Fuzzy logic system is used to select a new substitute for cluster head by considering residual energy and minimum distance node from faulty sensor node [16]. Saeed et.al. (2012) have proposed a fuzzy inference system based on probabilistic theory. In the suggested algorithm only one fault can be identified which is having different sensing value than expectation using variable rating method. If the result has substantial change from the rate variables of neighboring nodes then node is clear as faulty node [17]. Raja Al Kiyumiet. al. (2018) has suggested the fuzzy logic based routing algorithm for heterogeneous network. The main approach for the proposed model is to enhanced the lifetime of distributed network by reducing energy consumption based on a fuzzy logic approach [18]. Sai Krishna et. al. (2019) has suggested fuzzy based delay aware routing protocol. A node with greater residual energy, more buffer, available space with node, quality link and minimum distance acquires a chance to become the next suitable node in a data routing progression [19].

ZiQiet. al. (2015) has proposed a method to select cluster heads using fuzzy logic and circulate the $\mathrm{CH}$ selection decision to the base station. This method describes the centralized fuzzy fusion algorithm to perform three events such as cluster initialization, event detection and event warning phase based on distance, residual energy [20]. Pooja Jadavet. al. 
(2017) have explained fault recognition mechanism which identifies hardware faults like transmitter and receiver circuit fault [21].

\subsection{Problem Formulation}

The problem identified in many literature reviews is, if a node is not functioning properly then the node is considered as a faulty node or dead node and they cannot use a recovery mechanism to use the faulty nodes as much as they can. However, we can design a methodology to use the faulty node as per their status of faulty instead of declaring it as a fault node. This will improve the performance as well as increase the lifetime of wireless sensor networks. The purpose of the proposed work is to know the status of every node in advance and assign the work accordingly. The fuzzy logic approach is used to detect the faulty status of a node like normal node, node having less residual energy, node having different measured value than neighbor data and node not able to give response in terms of acknowledgement. In the proposed algorithm node fault is recognized in advance and according to that routing path has been decided. This helps to save energy because if we identified any fault in the routing phase then the whole network may collapse or create a delay in the data delivery process. Delay in data transmission cannot be tolerated in wireless sensor networks; otherwise the desired application will fail to take real time decisions. The aim of the proposed algorithm is to minimize the energy consumption by recognizing the fault in advance and enhancing the performance of the sensor network. Fuzzy logic is the most favorable solution to achieve the objectives. Many researchers prove that it gives a remarkable solution when it combines with wireless sensor networks Fuzzy logic uses linguistic parameters which prominently evaluates the performance of flexible parameters of the sensor network The significant finding from previous research motivates this work is the inadequate handling of faults in a routing path. In this work, fuzzy-based intelligent fault detection for inter-cluster and intra-cluster communication with energy recognition routing mechanism has been proposed to improve the lifespan of the sensor network.

\section{Fuzzy based fault recognition mechanism for heterogeneous wireless sensor network}

In this section a distributed network model and energy consumption mechanism has been described.

\subsection{Network Model}

A distributed sensor network in the proposed system consists of graph $G(V U A, E)$ as shown in Figure no. 1 where $V=\left\{V_{1}, V_{2}, V_{3}, \ldots, V_{n}\right\}$ is a set of $\mathrm{n}$ sensor nodes, $\mathrm{A}$ is an actuator node and $\mathrm{E}$ is a set of network links. Kautz graph concept is used to form a network. Actuator node is detecting events in the network. Distance between source and destination, hop count, residual energy, energy threshold value are considered as an evaluation parameter for analyzing the performance. Every node is having different residual energy value $(E i)$. Fuzzy fault count $(f f c)$ is calculated for each sensor node. Data transmission rate $(D r)$ is calculated based on fuzzy fault count $(f f c)$. A node $(V i)$ finding the efficient path by considering fuzzy logic pattern analysis $(\mathrm{Pi})$. The relevant abbreviations used in this paper are elaborated in Table. 1.

\begin{tabular}{|c|l|}
\hline Symbol & \multicolumn{1}{|c|}{ Description } \\
\hline$N$ & Total Number of nodes in the network \\
\hline$A$ & Actor node \\
\hline$E i($ res $)$ & Residual energy \\
\hline$\epsilon$ & Threshold Energy \\
\hline$D r$ & Data transmission rate \\
\hline$D s r$ & Distance between two nodes \\
\hline$F f c$ & Number of fault count \\
\hline$M F$ & Membership Function of input variable $X$ \\
\hline$P i$ & Fuzzy output value \\
\hline \multicolumn{2}{|c|}{ Tab 1. Represent Summary of Notation } \\
\hline
\end{tabular}

\subsection{Energy Consumption Model}

The amount of energy consumed by each sensor node during sensing operations, transmitting collected data to sink nodes and for receiving data uses radio model as explained in Ref. [22]. In the proposed energy consumption model, a free space model of multipath channels models is used. The selection of models is based on linking space between source and destination. The free space propagation model is used If the distance between source and sink node is less than the threshold value (do), otherwise, the multipath propagation model is used.

The Transmission energy $\mathrm{E}_{\tau}(m, d) \mathrm{E}_{\boldsymbol{r}}(m, d)$ to transmit m-bit message $(\mathrm{m})$ to distance $(\mathrm{d})$ for free space model is computed by Eq. (1) and for multi path model is calculated by Eq. (2) as given below. $E$ Thesec $E_{\text {Trescis }}$ the energy consumption for electronics device transmission and $\mathrm{E}_{T x \text {-amp }}$ is the energy consumption required to transmit data by amplifier.

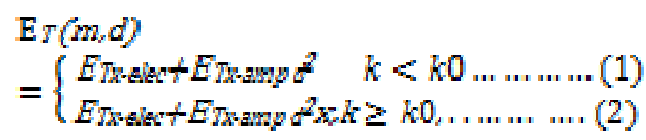

Whereas, the computation power for receiver sensor required by respective sensor node to receive $(\mathrm{m})$ bit message is calculated by Eq. (3).

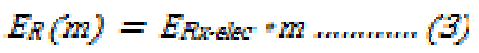

The energy consumption for sensing message (m) over a distance $(d)$ is represented by Eq. (4). If $(l)$ is the data generated by per event in time $(t)_{\sec }$ and $(\alpha)$ (a) is the total number of events happen in time $(t)_{\sec }$ then energy consumption for sensing procedure is computed by Eq.(5) and transmitting the sensing data is given by Eq. (6).

$$
\begin{aligned}
& E s(m)=E s \mathrm{sic} \cdot m
\end{aligned}
$$

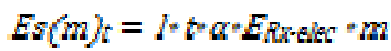

$$
\begin{aligned}
& E_{s}(m)_{t}=1=t \cdot \alpha=E_{\text {Rs alec }} \cdot m \\
& E_{s t}(m)_{t}=1+t-\alpha \cdot E_{T(n) d)}+E_{S(m)}
\end{aligned}
$$




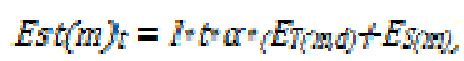

The remaining energy $E_{i}($ res $) E_{i}($ res $)$ of sensor node is evaluated by by Eq. (7) as given below:

$$
\begin{aligned}
& E i(r e s)=\operatorname{Eint}-\{(N t r: E t r-(N r e=E r-(N i=E i- \\
& (N g \times E g)\}
\end{aligned}
$$

Where, $\left(E_{\text {int }}\right)$ represents energy initialization of sensor nodes. (Ntr) denotes the number of data transmitted rounds, (Etr) represents transmitted energy. Whereas3.4 (Nre) indicates the number of rounds node receives the data, (Ere) is the total energy required for receiver operation. Similarly (Ni) and (Ns) represent how many times the node is in ideal state and sleep transition mode respectively. (Ei) is the energy dissipated in ideal state and (Es) represents energy consumption in sleep transition.

\subsection{Energy Aware Pre Fault Recognition Mechanism using Fuzzy Logic Approach (EAPFM)}

The working model of proposed fuzzy based energy aware pre fault recognition routing mechanism (EAPFM) and has been presented in Algorithm 1. The proposed algorithm calculates the fuzzy output values as a data transmission rate and best node for routing data. For pre fault detection, fuzzy fault count ( $f f c$ ) for every node is calculated to decide fault values like low, moderate or high in order to select the best routing path from source to destination. The proposed Fuzzy logic approach uses pattern analysis based on threshold value. Minimum and maximum threshold value for residual energy is considered. The parameters which are considered to update the fuzzy fault count are energy threshold value, sensing data value and distance. If the energy of a particular sensor is below the threshold value or sensed data is out of range of sensing range value then fault count $(f f c)$ is incremented by one. If fault count of the sensor node is above high range then that node is considered as permanent fault, otherwise it count as moderate or low,

accordingly data transmission rate is calculated to enhance the network lifetime and suitable best path for transmission. Following Metric are used to evaluate the performance in proposed algorithm:

a. Residual energy: This evaluation metric measures the instantaneous battery capacity of a node. According to battery level the routing decision has been taken in order to avoid permanent failure of a respective sensor node. Routing rules of the proposed mechanism considered this metric to identify a favorable path that has the adequate total energy capacity from source to destination [22].

b. Hop Count: From source to destination reference hop count and distance is calculated using Euclidean distance. While selective any node for identification of best suitable path distance and hop count is considered.

c. Sensor Sensing range: Based on sensing value of node routing decision has been taken. If a particular sensor node is giving value as per expectation then node is considered as faulty and not recommended for routing.

d. Fuzzy fault count: Fault count of every node is computed based on residual energy, hop count, sensing value of each node. Based on fuzzy fault count fault status, consider and take routing decisions and decide the data transmission rate in order to avoid permanent failure of nodes and save energy required for re-transmission.

\subsection{Fuzzy Logic System}

Fuzzy Logic System (FLS) is a non-linear pattern mapping [17] system. The pattern analysis is done with a fuzzy set that extends the conception of crisp sets. The fuzzy set has a membership function. The fuzzy systems are accompanying with the four components namely fuzzifier - it takes crisp inputs $(\mu(E), \mu(S))$ by using the membership functions, the fuzzy rules - which are expressed if- then rule, fuzzy inferences - the fuzzy inference associates the fuzzy rules to obtain an accumulated fuzzy output and finally the defuzzifier maps the fuzzy output back to a crisp data that can be used to take productive decisions [23].

In this proposed work, the input parameters for the fuzzy logic based fault detection prediction mechanism are residual energy, data aggregation value and hop count in terms of distance. The fuzzy input / output variables and linguistic factors are presented in Table (2). The fuzzy decision making rule set is described in Table 3. The linguistic factors low, medium, and high for residual energy and sensed data input variables are used. The fuzzy set that defines the distance input in terms of close, adequate and far. The fuzzy system output variable is to select the best node for routing data in an energy efficient way. The three linguistic variables low, weak and strong are considered for the fuzzy system output.

\begin{tabular}{|l|l|}
\hline Input/output variables & Linguistic variables \\
\hline Residual Energy & Low, Medium, High \\
Distance & Close, Adequate, far \\
$\begin{array}{l}\text { Data Sensing Value } \\
\text { Prediction of becoming next } \\
\text { node }\end{array}$ & Low, Medium, High \\
\hline
\end{tabular}

Tab. 2: Fuzzy Input / Output Variable and Linguistic variable

\begin{tabular}{|c|c|c|c|c|}
\hline $\begin{array}{c}\text { Residua } \\
\text { l Energy }\end{array}$ & $\begin{array}{c}\text { Hop } \\
\text { distance }\end{array}$ & $\begin{array}{c}\text { Data } \\
\text { Range }\end{array}$ & $\begin{array}{c}\text { Fuzzy } \\
\text { fault } \\
\text { Count }\end{array}$ & $\begin{array}{l}\text { Chance of } \\
\text { becoming } \\
\text { the next } \\
\text { node }\end{array}$ \\
\hline Low & Far & Low & High & Low \\
\hline Low & Far & Medium & High & Low \\
\hline Low & Far & High & High & Low \\
\hline Medium & Low & Low & High & Weak \\
\hline Medium & Low & Medium & Low & Weak \\
\hline Medium & Low & High & High & Weak \\
\hline High & Adequate & Low & Low & Strong \\
\hline High & Adequate & Medium & Low & Strong \\
\hline High & Adequate & High & Low & Strong \\
\hline
\end{tabular}

Tab. 3. Fuzzy decision-making rule set 
3.5 Algorithm 1: Energy aware pre fault recognition routing mechanism using fuzzy (EAPFM)

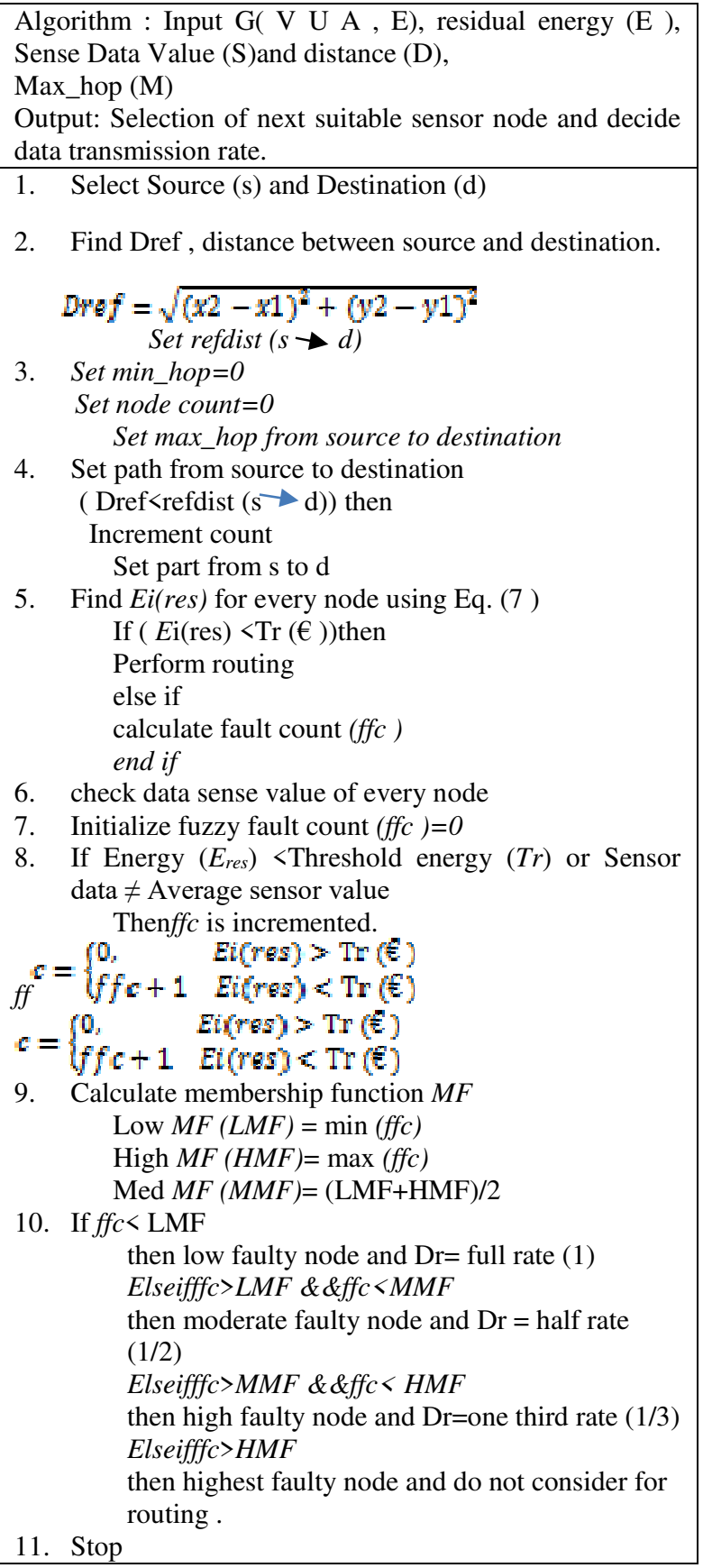

\section{Simulation Results}

The proposed model comprises a heuristic dispersed heterogeneous network with variable loads. In order to assess the efficiency of proposed algorithms various simulations have been carried out in NS2. In the network model heterogeneous sensor nodes are used with respect to battery capacity, transmission and reception data capacity and communication capacity is of duplex type. The evaluation parameters are considered for different combinations of fuzzy input i.e. threshold residual energy, sensing data and maximum hop count distance. The simulation parameters are shown in
Table 4 and experimental results by considering different scenarios have been presented in Table no 5, 6 and 7. The results are compared with some of the proactive routing protocol. We performed the simulation of the network based on two aspects. One is energy consumption of every node in the simulation period and secondly packet delivery ratio in case of node failure. The performance evaluation matrices for those two aspects are

1. Fuzzy fault count: The ratio of fault count of every node based on residual energy as well as data value in the network.

2. Data delivery rate: The ratio of data delivery rate based on status of fault like low, moderate or high.

3. Energy consumption of network: Energy consumed per event by each node in receiving and sending data packets throughout the simulation period.

In proposed fault recognition procedure, the routing decision has been taken using a fuzzy logic mechanism to minimize the energy consumption, reduce packet drop ratio and, reduce the delay and enhance the network lifetime.

\begin{tabular}{|l|c|}
\hline \multicolumn{1}{|c|}{ Parameter } & Value \\
\hline Network Area & $300 * 300$ \\
\hline Sensor node & $30-50$ \\
\hline Sensing Range & $10 \mathrm{~m}$ \\
\hline Threshold Energy & $200 \mathrm{~J}$ \\
\hline Maximum Packet Size & 1000 \\
\hline Node Initial Energy & $100 \mathrm{~J}$ \\
\hline $\begin{array}{l}\text { Transmitter circuit energy } \\
\text { consumption }\end{array}$ & $2 \mathrm{j} /$ bits \\
\hline Amplifier consumption & 1pj/bits \\
\hline
\end{tabular}

Tab. 4: Simulation parameters

\begin{tabular}{|c|c|c|c|c|c|}
\hline $\begin{array}{c}\text { No of } \\
\text { Commun } \\
\text { ication }\end{array}$ & AOSD & REFER & DPFDRM & FBFTN & EAPFM \\
\hline 5 & 15.52 & 65.091 & 48.98 & 22.136 & 18.136 \\
\hline 10 & 29.29 & 50.082 & 35.18 & 28.648 & 20.6 \\
\hline 15 & 37.63 & 92.748 & 112.88 & 143.43 & 73.43 \\
\hline 20 & 41.92 & 129.07 & 132.57 & 136.43 & 101.43 \\
\hline 25 & 56.19 & 225.28 & 153.64 & 241.7 & 141.1 \\
\hline 30 & 26.145 & 143.55 & 209.005 & 70.7474 & 68.245 \\
\hline 35 & 55.72 & 230.294 & 231.174 & 162.806 & 148.37 \\
\hline 40 & 45.399 & 46.978 & 53.565 & 68.584 & 49.545 \\
\hline 45 & 35.850 & 51.0293 & 53.565 & 55.369 & 40.345 \\
\hline 50 & 29.186 & 82.464 & 121.74 & 39.533 & 29.028 \\
\hline
\end{tabular}

Table 5: Simulation Results for delay (s)

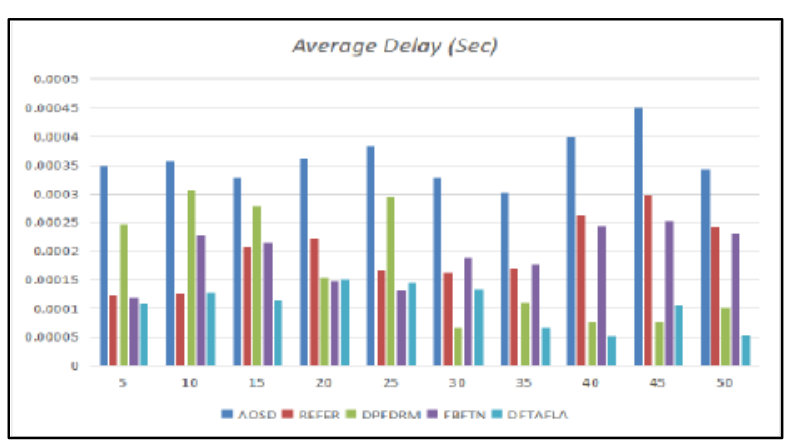


Fig 1: Comparison in delay for different number of communications

Table 5 describes the comparative performance results with a different number of communications in terms of delay. Figure 1 shows the graphical representation of average end to end delay of packet. The proposed mechanism uses three parameters: residual energy, data sensing value and distance. The traditional algorithm AOSD [25] , REFER [24] ,DPFDRM [22] and FBFTN [11] mechanism is based on residual energy and distance only. In the proposed distributed fault tolerance mechanism three parameters are considered like residual energy, data sensing value and distance using fuzzy logic. It can be observed that the proposed fuzzy logic-based fault tolerance mechanism performs better than the traditional algorithm.

\begin{tabular}{|c|c|c|c|c|c|}
\hline $\begin{array}{c}\text { No of } \\
\text { Comm } \\
\text { unicati } \\
\text { on }\end{array}$ & AOSD & REFER & DPFDRM & FBFTN & EAPFM \\
\hline 5 & 0.000349 & 0.000124 & 0.000246 & 0.000119 & 0.000109 \\
\hline 10 & 0.000356 & 0.000125 & 0.000307 & 0.000227 & 0.000127 \\
\hline 15 & 0.000328 & 0.000208 & 0.000278 & 0.000215 & 0.000115 \\
\hline 20 & 0.000362 & 0.000221 & 0.000154 & 0.000147 & 0.000150 \\
\hline 25 & 0.000383 & 0.000166 & 0.000296 & 0.000132 & 0.000145 \\
\hline 30 & 0.000329 & 0.000162 & 0.000066 & 0.000189 & 0.000134 \\
\hline 35 & 0.000302 & 0.000170 & 0.000110 & 0.000177 & 0.000065 \\
\hline 40 & 0.000399 & 0.000263 & 0.000077 & 0.000244 & 0.000051 \\
\hline 45 & 0.000450 & 0.000299 & 0.000077 & 0.000253 & 0.000107 \\
\hline 50 & 0.000343 & 0.000242 & 0.000101 & 0.000231 & 0.000053 \\
\hline
\end{tabular}

Table 6: Comparative Simulation Results for Energy Consumption $(\mathrm{J} / \mathrm{Sec})$

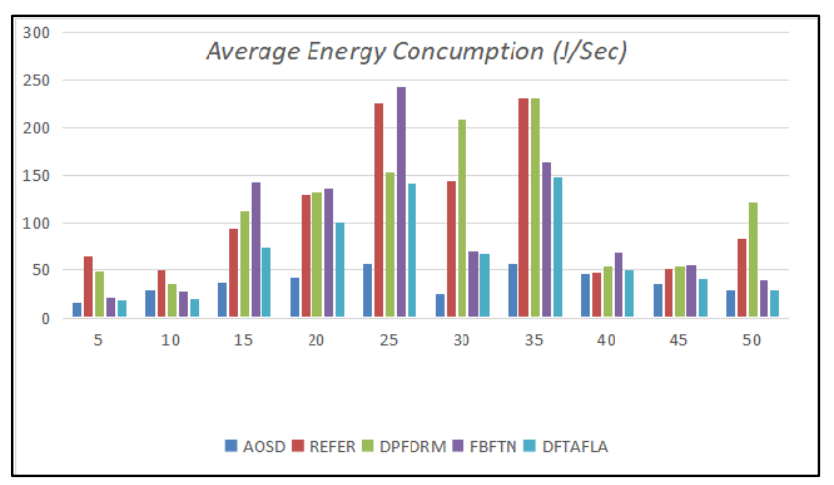

Fig 2: Energy Consumption Ratio in different number of communications

Table 6 describes the performance results within a different number of communications in terms of energy consumption. Figure (2) shows the graphical representation of average energy consumption ratio in different numbers of communications. The throughput and packet drop ratio from sender to receiver is shown in Table 7 and Table 8 respectively.

\begin{tabular}{|c|c|c|c|c|c|}
\hline $\begin{array}{c}\text { No of } \\
\text { Communication }\end{array}$ & AOSD & REFER & DPFDRM & FBFTN & EAPFM \\
\hline 30 & 100 & 97.99 & 99.33 & 98.25 & 99.638 \\
\hline 35 & 99.87 & 98.66 & 99.41 & 98.03 & 98.03 \\
\hline 40 & 100 & 97.701 & 99.51 & 98.189 & 100 \\
\hline 45 & 100 & 98.78 & 99.51 & 98.23 & 99.86 \\
\hline 50 & 100 & 98.32 & 98.93 & 97.65 & 99.79 \\
\hline
\end{tabular}

Table 7: Simulation Results for Throughput

\begin{tabular}{|c|c|c|c|c|c|}
\hline $\begin{array}{c}\text { No of } \\
\text { Communica } \\
\text { tion }\end{array}$ & AOSD & REFER & DPFDRM & FBFTN & EAPFM \\
\hline 30 & 208.53 & 202.7432 & 201.7738 & 202.807 & 201.9688 \\
\hline 35 & 206.555 & 204.981 & 203.4015 & 202.0021 & 204.26066 \\
\hline 40 & 208.472 & 203.0224 & 202.8201 & 202.461 & 206.0359 \\
\hline 45 & 208.570000 & 205.3214 & $\begin{array}{c}202.8201 \\
93\end{array}$ & 202.1017 & 206.616611 \\
\hline 50 & 205.0823 & $\begin{array}{c}203.14739 \\
1\end{array}$ & $\begin{array}{c}203.0240 \\
71\end{array}$ & 201.8629 & 201.345016 \\
\hline
\end{tabular}

Table 8: Simulation Results for Packet Delivery Ratio

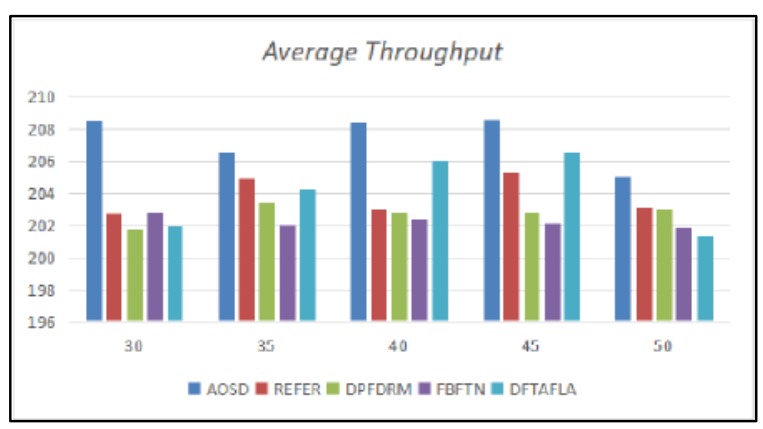

Fig 3: Comparative Throughput in different number of communications

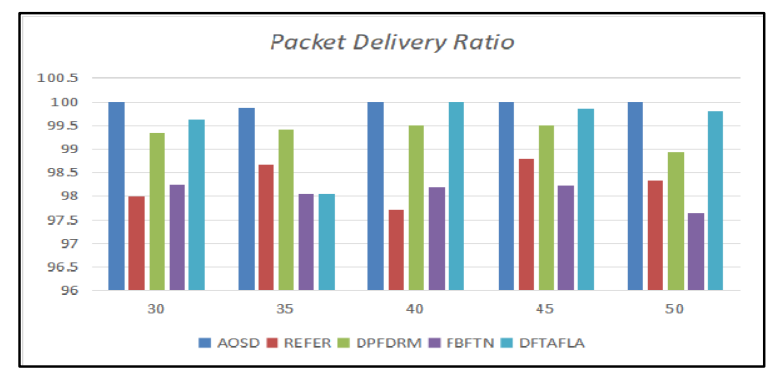

Fig 4: Packet Delivery Ratio in different scenario

Figure (3) and Figure (4) shows the comparison of throughput and packet delivery ratio of the proposed fuzzy based network mechanism with retransmissionbased approach. Due to retransmission more energy is consumed and retransmission is increased due to increased packet drop rate of every node in different communication in case of node failure. Proposed mechanism provides an efficient routing path in case of node failure. It uses fuzzy routing decision rules in case of node failure. This mechanism reduced the data transmission rate as per the status of fault like low, moderate or high. Most of the existing approach focuses only on permanent fault detection. 


\section{Conclusion}

Prolonging the network lifetime is the main motivation to design WSN routing protocols. To achieve this objective, the proposed mechanism has a novel approach based on fuzzy logic to reduce delay and energy aware pre fault recognition mechanism to identify the best suitable path for routing. Existing research mainly focuses on identification of permanent fault detection and consumes more energy in re-transmission. The proposed mechanism is implemented for a distributed sensor network and recognizes the faulty nodes based on residual energy, hop count distance and if a node sensed value has a significant difference with its neighbor values in a specific period; it would be detected as a faulty node. The proposed mechanism increased the rate of prediction of faulty nodes. The network performance is improved by considering fuzzy decision rules set to restrict the traffic in case of node failure. The extensive simulation result proved that the proposed mechanism reduces delay, packet drop ratio and energy consumption also as compared to AOSD, REFERS, DPFDRM and FBFTN mechanism. As a future research, we plan to consider mobile sink nodes for fault node data collection to investigate the performance.

Acknowledgement: Ms. Roshani Talmale likes to thanks both the research supervisors Dr. M. Nirupama Bhat and Dr. Nita Thakre for their time to time support, guidance and motivation. Also Roshani Talmale expresses her gratitude towards Head of Computer Science and Engineering Department Prof. Dr. Venkatesulu Dondeti for his support and cooperation.

\section{References}

[1] I.F.Akyildiz , I.H.Kasimoglu,"Wireless Sensor and Actor Networks: Research Challenges, " Ad Hoc Networks 2 (2004), Elsevier, pp 351-367

[2] I.F.Akyildiz, W.Su,Y et al, "Wireless Sensor Networks: a Survey," Computer Networks, vol 38 (2002) pp 393-422,

[3] K. Guleria and A. K. Verma, Comprehensive review for energy efficient hierarchical routing protocols on wireless sensor networks, Springer 2018, pp. 1159-1183.

[4] Z.W. Gao, and Cecati, C. "A survey of fault diagnosis and fault-tolerant techniques-Part I", IEEE Transactions, June 2015, vol. 62,pp 3757-3767.

[5] Y. Zhang and M/ Mukherjee, et al. "A Survey on Fault Diagnosis in WSN", 2018 IEEE. Translations, Volume 6, 2018, pp. 11349-11364.

[6] N. P. Srivastava and R. K. Srivastava, "Soft Computing Approaches to Fault Tolerant Systems", International Journal of Advanced Networking and Applications, 2014, Vol.5, Issue 6, pp 2096-2103.

[7] Mirjana M, Vladimir V and Vladimir M, "Fuzzy logic and Wireless Sensor Networks -A survey", Journal of Intelligent \& Fuzzy Systems 2014, Volume 27, pp 877-890,
[8] Deng, J., Han, R., Mishra, "INSENS: Intrusion-tolerant routing in wireless sensor networks", Computer Communications , 2006, Vol 29, pp 216-230

[9] Ganesan, D., S. Shenker, and D. Estrin, , "Highly-Resilient, Energy-Efficient Multipath Routing in Wireless Sensor Networks". Mobile Computing and Communications Review, Volume 5, No 4, pp 10-24.

[10] S. Javanmardi, A. Barati, S. J. Dastgheib and I. Attarzadeh, "A Novel Approach For Faulty Node Detection With The Aid Of Fuzzy Theory And Majority Voting In Wireless Sensor Networks", Int. Jour. of Advanced Smart Sensor Network Systems 2012, Vol 2, No.4, pp 1-10

[11] L. B. Bhajantri, "Fuzzy Logic Based Fault Detection in Distributed Sensor Networks", Int. Journal of Scientific Research in CSE, 2018 , Vol.6, Iss..2, pp.27-32

[12] Acharya, S., Tripathi, C.R., "A Reliable Fault-Tolerant ANFIS Model based Data Aggregation Scheme for Wireless Sensor Networks" , Journal of King Saud University Computer and Information Sciences (2017), Vol 32, Issue 6, July 2020, pp 741-753

[13] Acharya, S., \& Tripathy, C. R, 2018 "A Fuzzy Knowledge Based Fault Tolerance Mechanism for Wireless Sensor Networks", International Journal of Rough Sets and Data Analysis, Volume 5, Issue 1, pp.679-697

[14] P. Chanak, I. Banerjee, Fuzzy rule-based faulty node classification and management scheme for large scale wireless sensor networks, Expert Systems With Applications, 2015, Vol 45, pp 307-321

[15] K. M. Raghunath \& S. Thirukumaran, Fuzzy-based faulttolerant and instant synchronization routing technique in wireless sensor network for rapid transit system, Internet of Robotic Things For Smart Industrial Automation, Automatika, 2019, pp 1-8

[16] Farnaz Pakdel, Mansour Esmaeilpour Fuzzy Logic Method for Enhancement Fault-Tolerant of Cluster Head in Wireless Sensor Networks Clustering, TEM Journal. Volume 5, Issue 3, Pages 268-276

[17] Rogaia Mhemed et al., Energy Efficient Fuzzy Logic Cluster Formation Protocol in Wireless Sensor Networks", International Conference on Ambient Systems, Networks and Technologies, 2012, Vol.10, pp. 255 - 262, 2012.

[18] Al-Kiyumi, Raja, Foh, Chuan Heng, Vural, Serdar et al, "Fuzzy Logic-based Routing Algorithm for Lifetime Enhancement in Heterogeneous Wireless Sensor Networks" IEEE Transactions on green communications and networking.. pp. 517-532.2018

[19] S. Krishna M and R. R. Rout, "'Adaptive Fuzzy-Based Energy and Delay-Aware Routing Protocol for a Heterogeneous Sensor Network"', Journal of Computer Networks and Communications, Hindawi, Vol. 2019, 11 pages, 2019

[20] ZiQi H, Z Zhang,1 and Han-Chieh Chao,” A Cluster-Based Fuzzy Fusion Algorithm for Event Detection in Heterogeneous Wireless Sensor Networks", Journal of Sensor, Volume 2015, pp 1-11, 2015

[21] P. Jadav and Vinoth B.," Fuzzy Logic Based Faulty Node Detection in Wireless Sensor Network, International Conference on Communication and Signal Processing, IEEE, April 6-8, pp 390 - 394, 2017

[22] M. Younas et al.," FDRA: Fault detection and recovery algorithm for wireless sensor networks", Mobile Web and Intelligent Information Systems. Lecture Notes in Computer Science, Vol 10995, pp. 72-85. 2018 
[23] P. Chanak and I. Banerjee, "Fuzzy rule-based faulty node classification and management scheme for large scale wireless sensor networks," Expert Systems with Applications, vol. 45, pp. 307-321, 2016.

[24] Shen, H.Y., Li, Z., "A Kautz-based wireless sensor and actuator network for real-time, fault-tolerant and energyefficient transmission", IEEE Transactions on Mobile Computing, Vol 15, pp 1-16, 2015

[25] R. Gummadi, Todd Millstein, and Ramesh Govindan, "Declarative Failure Recovery for Sensor Networks-AOSD"' 07, March 12-16 2007.

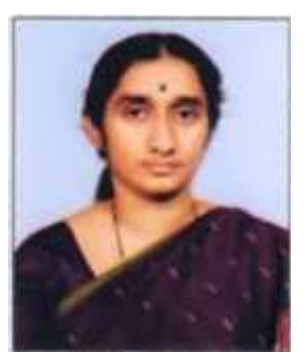

Nirupama Bhat Mundukur

Department of Computer Science, VFSTR deemed to be University, Guntur, and Andhra Pradesh, India. She received her Master's in Computer Applications and Ph.D

in Computer Science from Sri Padmavati Mahila Visvavidyalayam, Tirupati, in the area of Visual Cryptography in the year 2015. She is been associated with VFSTR deemed to be University, Vadlamudi, Guntur, Andhra Pradesh, India since 2007 and currently working as an Professor in the Department of Computer Science and Engineering. Her research interest includes Information Security, Data Analytics, Networks and Image Processing.

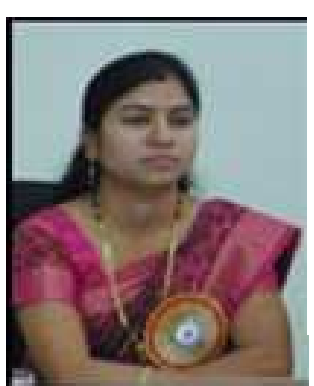

Roshani Talmale received the B.Tech degree in computer science from S.N.D.T. University, Mumbai in 2002 and $\mathrm{M}$ E. degree from RTM, Nagpur University, Nagpur, in 2014.

She is currently pursuing Ph.D. degree from Vignan's University, AP and working as a Assistant Professor in computer science department at S.B. Jain Institute of Technology, Management \& Research, Nagpur. 
Figures

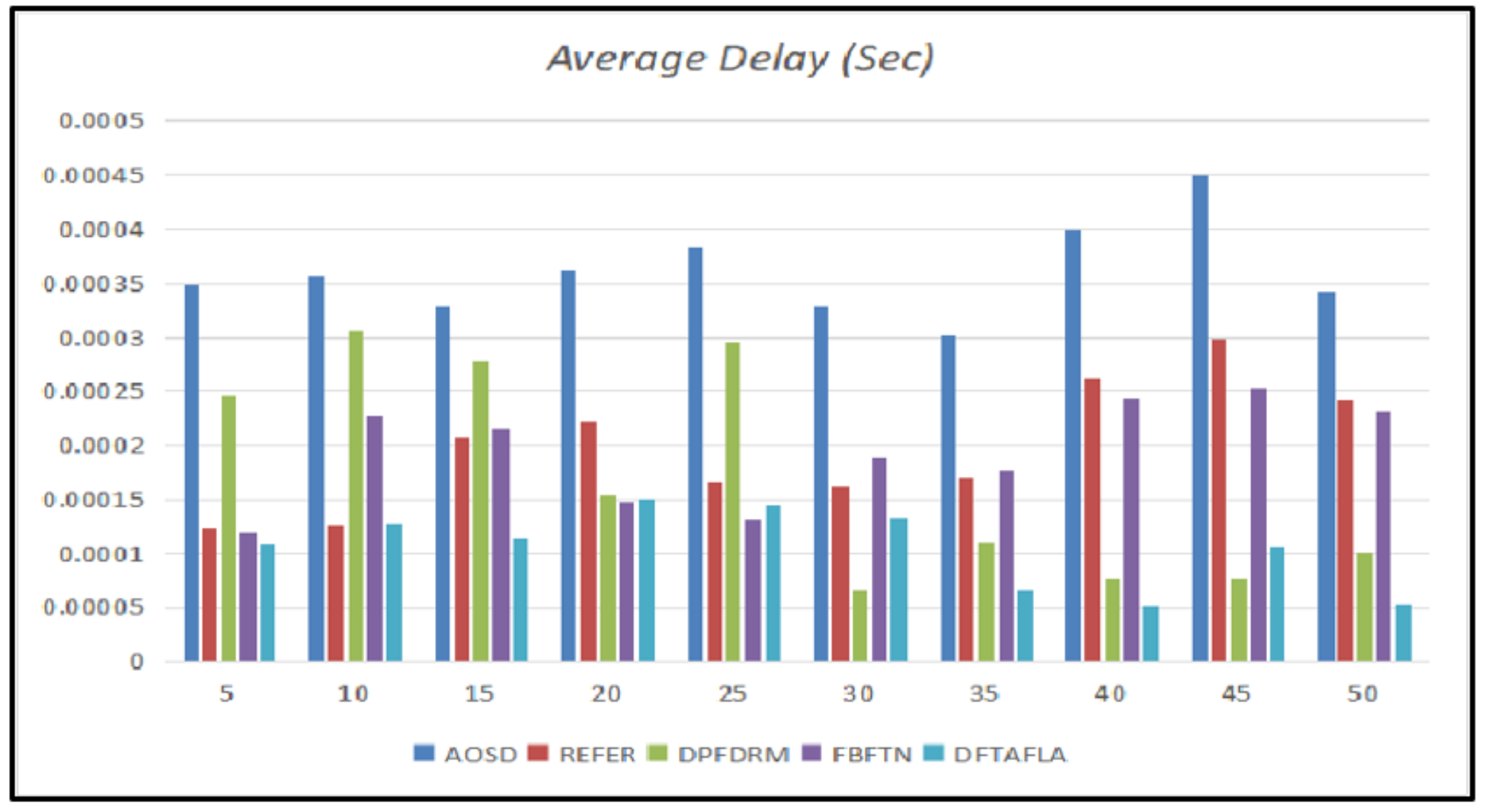

Figure 1

Comparison in delay for different number of communications 


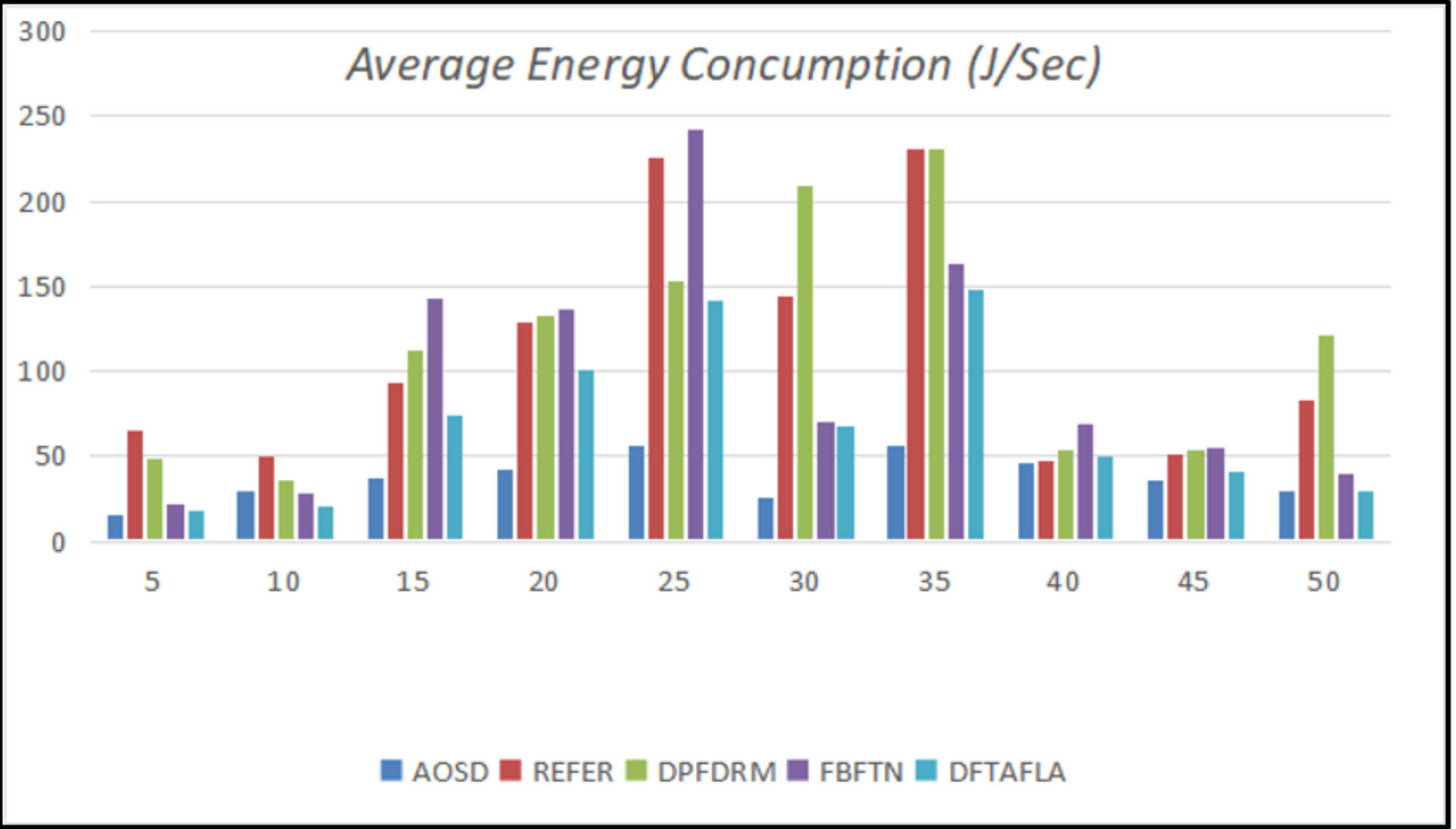

Figure 2

Energy Consumption Ratio in different number of communications

Average Throughput

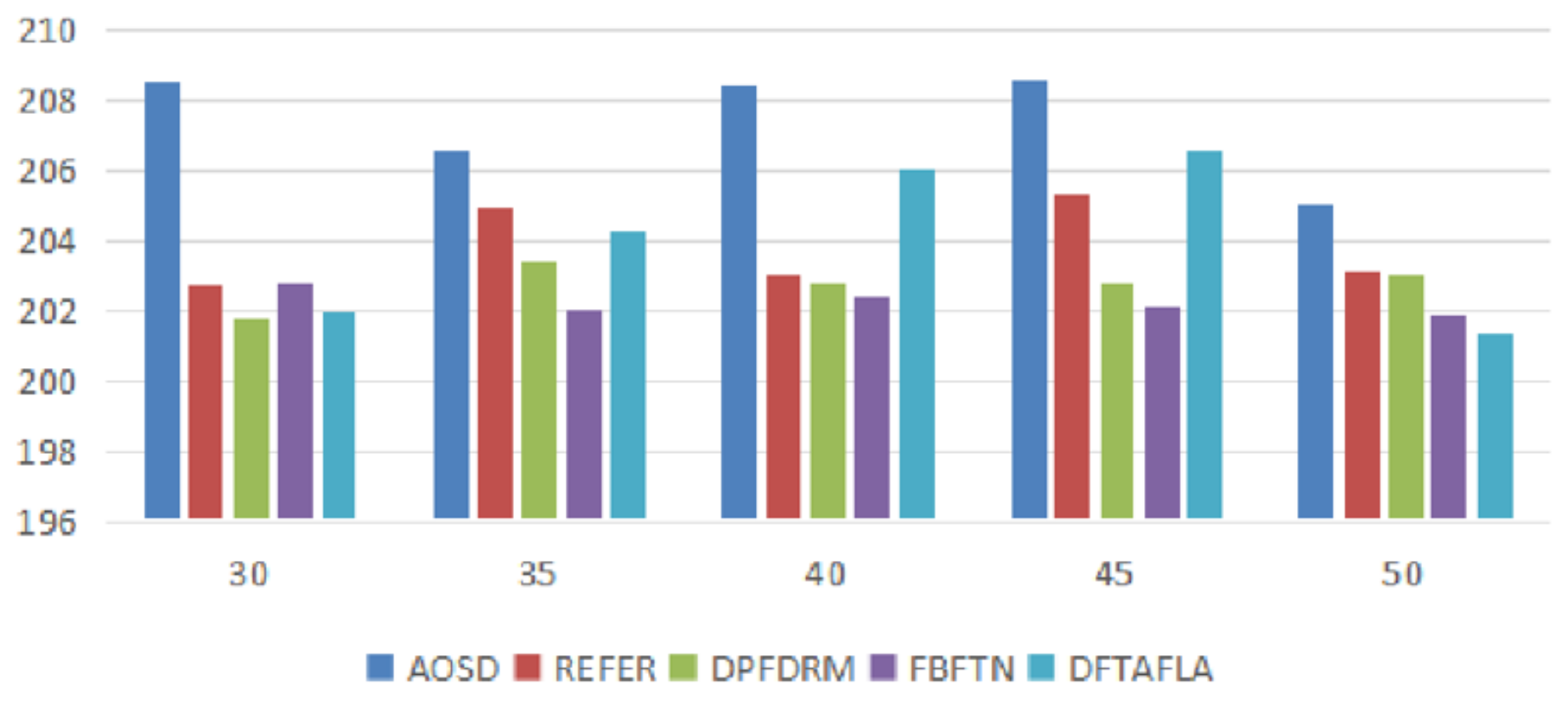

Figure 3 
Comparative Throughput in different number of communications

\section{Packet Delivery Ratio}

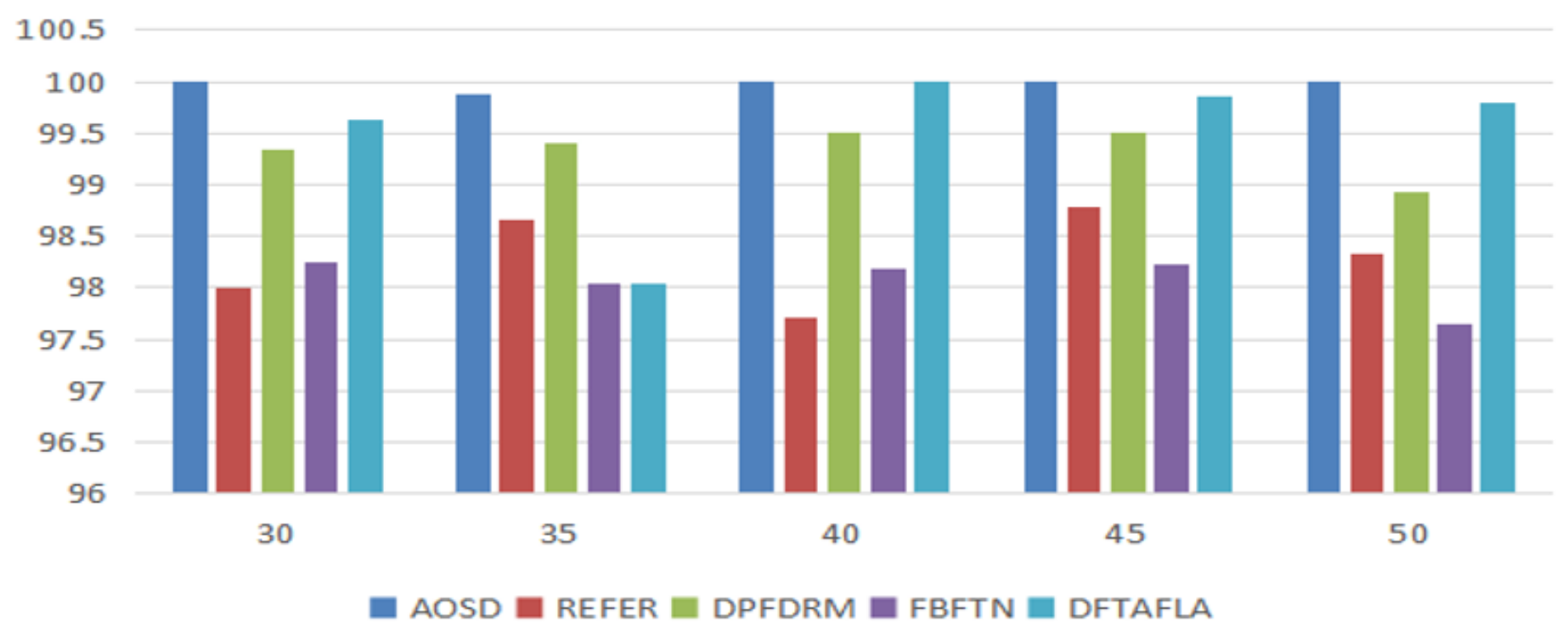

Figure 4

Packet Delivery Ratio in different scenario 